\title{
Evaluating the Quality of Medical Documentation at a University Teaching Hospital
}

\author{
Edward Ridyard, Elliot Street
}

United Kingdom

\begin{abstract}
A recent joint publication by the Royal College of Physicians and Royal College of Nursing raised concern regarding the variability in the organisation and quality of documentation during ward rounds [1]. The aim of the study was to evaluate the standard of medical documentation at a University Teaching Hospital. Retrospective manual analysis of patient's paper and electronic paper records (EPR) from the different specialties. Inclusion criteria included in-patient stay of more than two days and admission after 1st July 2013. A VTE assessment was available in $100 \%$ of patients. The plan and postoperative instructions were available in $100 \%$ of patients. Notes were documented contemporaneously in $75 \%$ of cases which increased to $80 \%$ in the second following by $89.11 \%$ in the third cycle. If a consultant was present on the ward round this was documented in $80 \%$ of cases in the first cycle. This subsequently increased to $90 \%$ in the second cycle and $100 \%$ in the third cycle. Overall the quality of medical documentation was of a reasonable standard but could be improved even further if we continue to document contemporaneously and name every person present at each patient encounter.
\end{abstract}

\section{Problem}

Accurate documentation in clinical medicine is vital for delivering safe patient care. Representative documentation is also important for research, audit, and quality improvement purposes. Medical notes also form the main body of the clinician's defence should a medicolegal issue arise. A recent joint publication by the Royal College of Physicians and Royal College of Nursing raised concern regarding the variability in the organisation and quality of documentation during ward rounds.

\section{Background}

In addition to the recent document produced by the Royal College of Physicians and Nursing, The National Health Service Litigation Authority (NHS LA) has produced a set of standards required for safe and effective patient care [1] with one section dedicated entirely to "Basic Record Keeping Standards". In addition, the Care Quality Commission's (CQC) Essential standards stipulate the need for accurate record keeping [2].

Following the introduction of Electronic Patient Records (EPR) to NHS trusts and the freedom of information available to patients, the need for transparent and clear documentation is even more important. The aim of the study was therefore to evaluate the quality of medical documentation in patients' notes across the different specialties based at the hospital.

\section{Baseline measurement}

As a comprehensive record of care, robust note-keeping is important for hospital service activity reporting as well as monitoring the performance of hospitals. Many of the causes of inaccurate clinical coding have been demonstrated to have been caused by the poor quality of medical notes[3,4]. In addition there is an evidence base to show that

structured records have beneficial effects on doctor performance and

patient outcomes [5].

\section{Design}

A set of criteria was compiled from a combination of the existing NHS LA standards and CQC Essential national standards [2,6]. In order to make the criteria as representative as possible the above standards were combined with the Oxford University Hospital Documentation Process guidelines, Oxford Radcliffe Hospital Records Procedure Version 3.0 [7]. A retrospective manual analysis of 20 sets of randomly selected patient paper and electronic notes was undertaken. The inclusion criteria for the study were admission after 1 st July 2013 and an inpatient stay of more than 48 hours. An inpatient stay of 48 hours was considered to be a significant inpatient stay were the documentation process could be adequately analysed.

The following criteria were assessed:

1. Venous thrombo-embolus (VTE) propylaxis assessment within 6 hours of admission

2. Plan to be clearly documented if problems identified

3. Postoperative instruction left if surgical procedure/interventional procedure performed

4. Most senior member of team/members of team present at consultation clearly documented

5. Documentation to be made contemporaneously when the patient was seen 


\section{Strategy}

PDSA Cycle 1

A set of criteria was compiled from a combination of the existing NHS LA standards and CQC Essential national standards along with Oxford University Hospital Documentation Process guidelines. Patients' notes were randomly selected and rigorously assessed according to criteria. Findings presented at the departmental audit meeting. At the audit meeting awareness was increased of the need to document contemporaneously and to document most senior person present on ward round.

PDSA Cycle 2

6 months following intervention. Findings presented internationally at Nordic Orthopaedic Federation Conference, Helsinki, Finland. Departmental staff made further aware of the importance of medical documentation.

PDSA Cycle 3

One year following intervention further notes analysed to assess for further improvement.

\section{Results}

A VTE assessment was performed within 6 hours of admission for $80 \%$ of patients although a VTE assessment was available in $100 \%$ of patients. The plan and postoperative instructions were available in $100 \%$ of patients.

Notes were documented in retrospect in $25 \%$ of cases and in all of these cases it was not specified by what time period in retrospect the entry was made. In the second cycle the entries were documented contemporaneously or specified by how much in retrospect in $80 \%$ of cases and this subsequently increased to $89.11 \%$ in the third cycle.

If a consultant was present on the ward round this was documented in $80 \%$ of cases in the first cycle. This subsequent increased to $90 \%$ in the second cycle and $100 \%$ in the third cycle.

\section{Lessons and limitations}

One of main the limitations of the study was assessing exactly which of the junior clinicians were present on the ward round. Although it would appear that a Junior Doctor sees a patient $63.00 \%$ of the time it is most likely the figure is much higher than this. It is custom for the most senior person on the round to have their name in the title with the scribe signing off the entry at the end. This assumes only two people were present on the round which is rarely the case. In situations where a patient's case is reviewed or issues of clinical negligence arise it is imperative to know exactly who was present at each patient encounter in order to pinpoint exactly who was responsible for that patient's care at that specific moment. This can be remedied by every person who was present on the round including Nurse team and Allied Healthcare Professionals being mentioned at some point in the medical entry.

Although being more convenient and arguably easier to locate specific entries EPR presents a new problem in that it is difficult to assess whether a note was made contemporaneously. This is because unless it is specified by how much in retrospect a patient was seen, it is impossible to determine if the patient was seen at the actual time that EPR automatically assigned to the note. This could be addressed by the clinician making the entry specifying in the notes (as is done manually in paper notes) the time at which the patient was actually seen in the title of the note.

\section{Conclusion}

Overall the quality of medical documentation was of a reasonable standard. The plans and postoperative instructions were clearly documented in all patients who underwent surgery/a procedure as were all of the VTE assessments. To improve the quality of medical documentation further it is necessary for the person making the entry to specify exactly who is present at each patient encounter and if a note is made in retrospect to specify by how much in retrospect the entry is made.

\section{References}

1. Royal College of Physicians and Royal College of Nursing. 2010. Ward Rounds in Medicine: Principles for Best Practice. London.

2. Care Quality Commission (CQC) website. CQC Essential Standards of Quality and Safety, Outcome 21. http://www.cqc.org.uk/sites/default/files/media/documents/g ac -

_dec_2011_update.pdf (date accessed: 30th March 2014).

1. Royal College of Physicians, 2007. Hospital Activity Data. A guide for

Clinicians. London.

1. The Audit Commission, 2008. PbR Data Assurance Framework

2007/2008: Findings from the first year of the national clinical coding

audit programme. London.

1. Mann R, Williams J. 2003. Standards in medical record keeping. Clinical

Medicine; 3:329-32.

1. NHS Litigation Authority (NHS LA) website. NHS LA Standards 2013-14, section 1.8. http://www.nhsla.com/Pages/Publications.aspx 
library=safety\%7cstandards. (date accessed: 15th February 2015).

1. Oxford University Hospitals (OUH) Trust Website. OUH Documentation Process and Oxford Radcliffe Hospitals Records Procedure Version 3.0.

http://ouh.oxnet.nhs.uk/SafetyQualityRisk/Document\%20Library/Cli nical\%20Audit/Clinical\%20Audit\%20Committee/CAC\%202013\%20 (01)\%20January/CAC\%200113.11\%20TW\%20Record\%20Keeping \%20Clinical\%20Audit\%20Final\%20Report\%2012.12.2012_D1277.p df (date accessed:15th February 2015).

\section{Declaration of interests}

Nothing to declare

\section{Acknowledgements}

Elliot Street, Charlotte Williams 